\title{
Die Umsetzung in der Praxis braucht Zeit
}

\begin{abstract}
Diabetologen sind sich einig: Neue orale Antidiabetika sollten aufgrund der dokumentierten kardiovaskulären Sicherheit, des fehlenden intrinsischen Hypoglykämierisikos und günstiger Zusatzeffekte gemeint sind v.a. DPP-4- und SGLT-2-Hemmer - ältere Substanzen ohne entsprechende Daten und mit Hypoglykämierisiko - in erster Linie Sulfonylharnstoffe - zukünftig ersetzen. In der Praxis verläuft die Umsetzung dieser Empfehlung aber zögerlich.
\end{abstract}

„Ich sehe keinen Grund mehr für den Einsatz von Sulfonylharnstoffen (SH) außer, ökonomisch unschlagbar zu sein“, resümierte Prof. Thomas Forst aus Mainz seinen Vortrag zu sicherheitsrelevanten Daten der SH. Da deren Sicherheit nicht dokumentiert sei, würden SH heute nicht mehr zugelassen, betonte der Diabetologe. In fast allen Beobachtungsstudien zeigte sich bei retrospektiver Analyse der SH-Daten eine signifikant erhöhte der Gesamt- und kardiovaskuläre Mortalität. Auch in der UKPD-Studie, die oft als Beleg für die Sicherheit der SH genutzt werde, sei die Mortalität bei der Kombitherapie SH plus Metformin signifikant erhöht gewesen. Und in der kontrollierten ADOPT-Studie ergaben sich Hinweise auf Erhöhung der kardiovaskulären Mortalität mit SH (Kahn SE et al. NEJM 2006; 355:242743). In einer Metaanalyse von 8 RTs (Hemmingsen B et al. CMAJ Open 2014;2:E162-75), die zu einem neutralen Ergebnis kam, seien geringe Ereignisraten und die Therapiedauer von nur 6-12 Monaten zu beachten, so Forst. Als Gründe für die Mortalitätserhöung mit SH werden Aktivierung kardialer Kanäle, Hypoglykämie, Proinsulinämie und adipogene Effekte diskutiert. Die antiglykämische Wirkung von SH halte zudem meist nur 2-3 Jahre an. Angesichts der verfügbaren sicheren Alternativen sollten SH nur mit äußerster Sorgfalt und unter Berücksichtigung des individuellen Risikos eingesetzt werden.

Bei Inkretinmimetika war ein evtl. erhöhtes Risiko für Pankreatitis und Pankreas-Ca im Gespräch. Diese Sorgen sind für DPP-4-Hemmer durch Studien wie SAVOR-TIMI-53 (Saxagliptin) und TECOS (Sitagliptin) weitgehend ausgeräumt. Es gab kein Signal für vermehrte Pankreasereignisse, so Prof. Rüdiger Göke, Marburg. Durch den Wirkmechanismus könne ein Pankreasrisiko auch nicht erklärt werden. Auch für s.c. applizierte GLP-1-Agonisten sei die „bisherige Studienlage mehr als überzeugend“. Es hätten sich keine Hinweise für mehr Pankreatitiden oder Pankreaskarzinome ergeben. Durch große Endpunktstudien zur kardiovaskulären Sicherheit von DPP-4-Hemmern wurde zudem der durch SAVOR-TIMI-53 geschürte Verdacht für ein evtl. erhöhtes Risiko für Herzinsuffizienz (HI) mit Gliptinen entkräftet. Dagegen entwickelten Typ-2-Diabetiker ohne kardiovaskuläre Ekrankung in einer Untersuchung der American Diabetes Association mit SH signifikant öfter HI als mit DPP-4-Hemmern, so Göke. Bei Patienten mit kardiovaskulären Erkrankungen wurden hier keine Unterschiede gefunden.

Sehr gut ist die Datenlage zu SGLT-2-Hemmern. Sie erhöhen nicht das Hypoglykämierisiko und beeinträchtigen nicht die

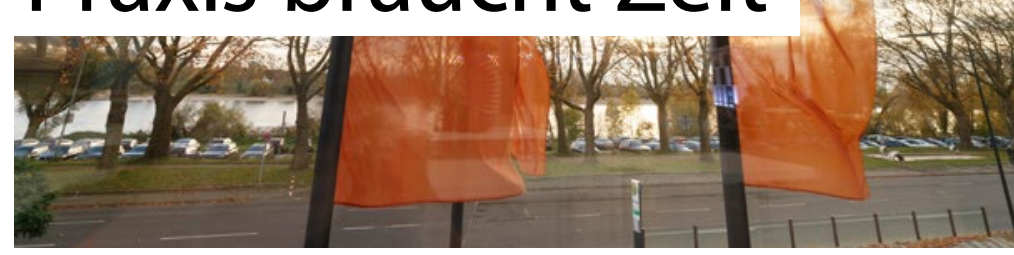

Nierenfunktion, so Prof. Jochen Seufert, Freiburg. Allerdings nimmt die Wirksamkeit der Substanzen, die die Glukoseausscheidung erhöhen, mit der Nierenfunktion ab. Als günstige Begleiteffekte nannte Seufert eine Gewichtsreduktion von ca. 3-4 $\mathrm{kg}$, vermutlich durch verminderte Glukoserückresorption, und die Reduktion des Blutdrucks um ca. 2-3mmHg syst.. Eine klassenspezifische Nebenwirkung sind mykotische Genitalinfekte, meist Candida, die laut Seufert bei ca. $4 \%$ der Patienten aufträten und topisch gut zu behandeln seien. Gefährdet seien v.a. Patienten mit positiver Anamnese für Genitalinfekte. Weniger deutlich sei mit SGLT-2-Hemmern die Erhöhung des Risikos für Harnwegsinfekte. Infolge der Diurese verringert sich das Plasmavolumen und erhöht sich evtl. der Hämatokrit. Untersucht werden von FDA und EMA derzeit > 100 Spontanberichte von diabetischer Ketoazidose (DKA) unter SGLT-2-Hemmern ohne drastisch erhöhte Blutzuckerwerte. Rund ein Drittel von ihnen traten bei Off-label-Therapie von Typ-1-Diabetikern auf. Es wurde bisher kein kausaler Zusammenhang belegt, so der Diabetologe. In der EMPA-REG-Outcome-Studie zu Dapagliflozin bei mehr als 7.000 Typ-2-Diabetikern, der ersten Studie mit Daten zur Mortalitätsreduktion durch ein OAD, wurden in der Verumgruppe 4 Fälle einer DKA vs. 1 Placebo berichtet (Zinman B et al. NEJM 2015;373:2117-28). Dem unter Umständen erhöhten Ketoazidoserisiko unter Gliflozinen wird inzwischen mehr Aufmerksamkeit gewidmet. Profitieren tun von SGLT-2-Hemmern nach Angaben von Seufert v.a. Diabetiker mit Übergewicht, Hypertonie oder hohem Hypoglykämierisiko sowie mit hohem kardiovaskulären Risiko. Nicht sinnvoll sei der Einsatz bei Niereninsuffizienz ( $<45 \mathrm{ml} / \mathrm{min}$ ) oder hohem Risiko für Genitalinfektionen, Hypovolämie oder Ketoazidose.

Und wie sieht die Versorgungsrealität aus? Auf Antidiabetika entfielen 2014 Kosten von ca. 1,8 Mrd. Euro, davon 31\% aufOAD, so PD Dr. Rainer Lunderhausen, Erfurt. Die SH-Verordnung sei in den letzten Jahren stetig gesunken, die von DPP-4- und SGLT2-Hemmern kontinuierlich gestiegen. Aber noch immer liegt der Marktanteil von SH (Tagestherapiedosen, TD) bei knapp 20\%, damit fast so groß wie der von DPP-4-Hemmern. SGLT-2-Hemmer haben bisher nur wenige Prozent Marktanteil. Unter den Top-Ten der Antidiabetika nach Verbrauch 2014 (inkl. Insulin) war mit Sitagliptin (kombiniert mit Metformin Platz 6, Monotherapie Platz 8) nur ein neues OAD vertreten (Arzneimittel-Atlas). Auf Platz 1 mit großem Abstand: Metformin (knapp 600 Mio. TD, auf Platz 2 immer noch Glimepirid (255 Mio. TD). Beim Umsatz sieht es klar anders aus. 2014 entfielen fast 80\% der Gesamtkosten für OAD auf DPP-4-Hemmer, jeweils ca. $6 \%$ auf Metformin und SGLT-2-Hemmer, knapp 4\% auf SH. Roland Fath

Quelle: DDG-Herbsttagung 2015. 6.-7. 11.2015, Düsseldorf 